\title{
Sexually transmitted infections and use of contraceptives in women living with HIV in Denmark - the SHADE cohort
}

Kristina Thorsteinsson ${ }^{1 *}$ D , Steen Ladelund ${ }^{2}$, Merete Storgaard ${ }^{3}$, Frederikke Falkencrone Rønsholt ${ }^{4}$, Isik Somuncu Johansen ${ }^{5}$, Gitte Pedersen ${ }^{6}$, Lars Nørregård Nielsen ${ }^{7}$, Jesper Bonde ${ }^{8}$, Henrik Westh ${ }^{9,10}$, Niels Obel ${ }^{4,9}$, Terese L. Katzenstein ${ }^{4,9}$ and Anne-Mette Lebech ${ }^{1,9}$

\begin{abstract}
Background: No Danish guidelines for screening of sexually transmitted infections (STIs) in women living with HIV (WLWH) exist, except for annual syphilis testing. Drug-drug interaction between hormonal contraceptives and some types of highly active antiretroviral therapy (HAART) occurs. We assessed prevalence of STIs, contraceptive choices and predictors of condom use in a cohort of WLWH in Denmark.

Methods: WLWH consecutively enrolled during their outpatient visits from 2011 to 2012 . Gynaecological examination and an interview were performed at entry and 6-month follow-up. Inclusion criteria were HIV-1 infection and $\geq$ 18 years of age. Exclusion criteria were pregnancy, alcohol- or drug abuse impeding adherence to the protocol. At entry, participants were tested (and where appropriate, treated according to guidelines) for Chlamydia trachomatis, Neisseria gonorrhoeae, syphilis, and herpes simplex (HSV-1 and HSV-2). At follow-up, predictors of condom use were estimated in sexually active WLWH.

Results: In total, 334 of the 1,392 eligible WLWH in Denmark were included (median age and HIV duration: 42.5 and 11.3 years). Chlamydia trachomatis was present in four individuals (1\%), and six (2\%) tested positive for HSV-2 by PCR. None were positive for Neisseria gonorrhoeae, HSV-1 or had active syphilis. At follow-up, 252 (76\%) participated; 168 (70 \%) were sexually active. Contraceptives were used by 124 (75\%); condoms were preferred (62\%). Having an HIV-negative partner predicted condom use (adjusted OR 3.89 (95\%Cl 1.4910.11)). In the group of participants of reproductive age without pregnancy desires $13 \%$ used no birthcontrol. Possible drug-drug interaction between hormonal contraceptives and HAART was found in 13/14 WLWH receiving both kinds of medication.

Conclusion: The prevalence of STIs in WLWH in Denmark was low. The need for annual STI screening is questionable. Condoms were preferred contraceptives, especially in WLWH with an HIV-negative partner. In this cohort, $13 \%$ of WLWH of reproductive age were at risk of unintended pregnancies due to lack of birth-control. Finally, in the subgroup of WLWH receiving both hormonal contraceptives and HAART possible drug-drug interactions could occur.
\end{abstract}

Keywords: Women living with HIV, Sexually transmitted infections, Screening, Contraceptive use, condom use, Hormonal contraception, Sexual activity

\footnotetext{
* Correspondence: kristina.thorsteinsson@gmail.com

'Department of Infectious Diseases, Hvidovre, Copenhagen University

Hospital, Copenhagen, Denmark

Full list of author information is available at the end of the article
} 


\section{Background}

New attitudes towards condomless sex in people living with HIV-1 infection (PLHIV) have emerged as a result of among others the HPTN 052 trial that found reduced rates of sexual transmission of HIV in patients with early initiation of highly active antiretroviral therapy (HAART) [1] and the interim analysis of the PARTNER study reporting zero linked HIV transmissions from condomless sex within mixed-HIV-status couples, when the partner living with HIV had a viral load below 200 copies/mL [2]. Accordingly, sexual HIV transmission is thought to be negligible in patients adherent to HAART, with a suppressed viral load, and without sexually transmitted infections (STIs) [1, 2].

Increased incidences of STIs - serving as a proxy of condomless sex, especially among men who have sex with men (MSM) [3, 4] - are a risk to health and facilitate acquisition and transmission of $\operatorname{HIV}[3,5]$. Yet, data regarding the prevalence of STIs among women living with HIV (WLWH) are scarce [4, 5], and most existing studies focus on women newly diagnosed with HIV [5]. Guidelines from the European Aids Clinical Society (EACS) recommend that screening for STIs should be offered to all sexually active PLHIV at time of HIV diagnosis, annually thereafter or at any time STI symptoms are reported [6]. In Denmark, however, no guidelines for routine STI screening in PLHIV exist, except for annual syphilis testing [3]. Whether STI screening is needed in WLWH is unknown.

The trend towards condemless sex yields for alternate means of contraception. Most WLWH are of childbearing potential $(10 ; 11)$, which makes counselling on family planning and use of contraceptives a most relevant issue.

This article reports the first data from the Study on HIV, cervical Abnormalities and infections in women in Denmark (SHADE). Several gaps in evidence in caring for WLWH have been identified such as sexual health, gynaecological diseases and contraceptive use [7, 8]. The aim of the present study was to evaluate the prevalence of STIs in a cohort of WLWH and to assess whether routine screening for STIs in WLWH in Denmark is needed. Moreover, we describe sexual activity, contraceptive choices, and predictors of condom use in a cohort of WLWH in Denmark with free access to healthcare and HAART.

\section{Methods}

\section{Setting}

Denmark has a population of 5.6 million [9] and an estimated HIV prevalence among adults of $0.1 \%$ [10]. Medical care, including HAART, is tax-paid and provided free-of-charge to all PLHIV. Treatment of HIV is restricted to eight specialized medical centres. Six of these centres (treating $97 \%$ of Danish PLHIV) (Copenhagen
University Hospital, Hvidovre, (HVH), Skejby, Aarhus University Hospital (AUH), Copenhagen University Hospital, Rigshospitalet ( $\mathrm{RH})$, Odense University Hospital (OUH), Aalborg University Hospital (AAUH), and Nordsjællands Hospital Hillerød $(\mathrm{HIH})$ ) participated in SHADE (see below).

\section{The SHADE cohort}

The SHADE cohort is an ongoing, multicentre, prospective, observational cohort study of WLWH in Denmark attending regular outpatient care for their HIV infection. Study participants were consecutively enrolled during their outpatient visits from 1 February 2011 to 1 February 2012 and followed-up after 6 months. The study focuses on STIs, contraceptive choices, sexual activity, cervical abnormalities and cancer, human papillomavirus (HPV) infection, HIV disclosure and other aspects of living with HIV as a woman.

\section{Study population}

WLWH in Denmark attending care in one of the Danish HIV centres. Inclusion criteria were HIV-1 infection and $\geq 18$ years of age. Exclusion criteria were pregnancy, alcohol- or drug abuse impeding adherence to the protocol.

\section{Interview survey}

Standardized interview questions were developed in consultation with clinical specialists and a statistician and appropriate revisions were made after a pilot study including ten HIV-negative volunteers. Questions were in Danish and closed-ended (Additional file 1 and Additional file 2; questionnaires in English). Double manual data entry of the questionnaires was performed, using the EpiData Entry program [11].

Trained doctors performed the standardized interviews regarding weight/height, tobacco and alcohol consumption, lifetime sexual partners, history of herpes and condyloma, symptoms from the lower abdomen, HPV vaccination status, adherence to HAART, and use of contraceptives.

At 6-month follow-up a similar interview regarding marital status, partner's HIV status, sexual activity within the past 6 months, practice of anal sex, and possible reasons for no condom use was performed.

\section{Registries}

\section{The Civil Registration System (CRS)}

The CRS is a national registry of all Danish residents [12]. A 10-digit personal identification number (PIN) is assigned to each individual at birth or immigration. The PIN was used as a linkage to the Danish HIV Cohort Study (DHCS). 


\section{Danish HIV Cohort Study (DHCS)}

The DHCS is a prospective, observational, nationwide cohort study of all PLHIV seen at the Danish HIV clinics since 1January 1995 [13]. Data collection is ongoing, with continuous enrolment of newly diagnosed PLHIV. The database is updated annually and contains extensive data on demographics, date of HIV diagnosis, laboratory results etc.

\section{Microbiological testing Chlamydia trachomatis (CT) and Neisseria gonorrhoeae (NG)}

Samples were analysed according to local guidelines at the local departments of microbiology. At HVH, a BD ProbeTec ET Wet Swab was used for detection of $C T$ and NG on oral, cervical, anal and first-void urine specimens (for the latter using the BD ProbeTec Urine Preservative Transport Kit (UPT)). At AAUH and RH, cervical and urethral specimens were examined for $C T$ with a COBAS $^{\oplus}$ Taqman $^{\oplus} 48$ analyzer (Roche Molecular Diagnostics, Indianapolis, IN, USA). Further, cervical, anal, urethral and oral specimens were obtained for culture of NG with charcoal swabs and transported in Stuart medium (SSI Diagnostica, Hillerød, Denmark). On specimens from AUH, OUH and $\mathrm{HIH}$, the GenProbe Panther Aptima Combo2 test (San Diego, USA) was used for detection of $C T$ and $N G$.

\section{Syphilis}

Until 1 July 2011 [14], syphilis serology was centralised at Statens Serum Institut (SSI) (the National Institute for Health Data and Diseases Control). Two non-treponemal tests were used; Wassermann's reaction (WR) was done with a complement fixation technique, and rapid plasma reagin (RPR) was determined by agglutination [3]. Treponemal tests were performed on seroreactive samples [3]. After 1 July 2011 syphilis serology was decentralised to the local departments of microbiology performing a treponemal test detecting specific IgG and/or IgM antibodies to Treponema pallidum using the ADVIA Centaur ${ }^{\bullet}$ Syphilis assay (Siemens, US), the Syphilis TPA, from Vitros' Immunodiagnostics, Ortho Clinical Diagnostics (High Wycombe, UK) or the Architect Syphilis TP (Abbott Japan, Japan: AST). If the antibody test was positive, nontreponemal tests (WR/RPR) were sent to SSI for confirmation. Patient files were examined regarding prior syphilis infection and treatment.

\section{Herpes Simplex 1 and 2 (HSV-1 and HSV-2)}

Diagnosis of HSV-1 and 2 was conducted at Department of Pathology, HVH. WLWH were swabbed once at entry and cervical samples were examined by the CLART Entherpex PCR (Genomica, Madrid, Spain). DNA extraction was done using the MagNAPure LC96 (Roche
Molecular Systems, Rotkreuz, Ch). Samples showing an invalid outcome were retested, and the second result was considered definitive.

\section{HIV RNA}

Undetectable viral load was defined as a plasma HIV RNA load of $<40$ copies $/ \mathrm{mL}$, which was the highest level of sensitivity for testing in the observation period.

\section{Ethics, consent and permissions}

At entry, written and oral informed consent was obtained from all participants. The study and the DHCS were approved by the Danish Data Protection Agency (2015-231-0126, 2012-58-0004 and 2012-41-0005). Further, the study was approved by the Danish Regional Committee on Health Research Ethics (approval numbers: H-3-2010-119 and H-2-2014-102).

\section{Statistical analyses}

Continuous variables were summarized as median and interquartile ranges (IQR) and compared using Wilcoxon rank sum test. Categorical variables were reported as counts and percentages and compared by chi-square test or Fisher's exact test as appropriate.

To calculate age and follow-up time for participants in the study and WLWH in the remaining DHCS 1 February 2012 (the last possible day of inclusion) was defined as day of inclusion.

Multivariate logistic regression analyses were performed to identify predictors of condom use in sexually active WLWH attending 6-month follow-up. Odds ratios (ORs) and $95 \%$ confidence intervals (CI) were estimated and adjusted for seven candidate predictor variables chosen a priori, including age, race, HIV-RNA at inclusion, number of lifetime sexual partners, marital status, partner's HIV status, and self-reported HSV infection prior to inclusion. To control for repeated testing, a combined $p$-value was estimated for variables spending more than one degree of freedom in the logistic regression.

Individuals with missing explanatory values were excluded from the multivariate regression analyses. The validity of the model was tested using the Hosmer and Lemeshow Goodness-of-Fit Test.

SAS statistical software version 9.3 (SAS Institute Inc., Cary, NC, USA) was used for data analysis and $p$-values $<0.05$ (two-sided) were considered statistically significant.

\section{Results}

Of the 1,392 WLWH alive and $\geq 18$ years of age in the DHCS, 334 (24\%) consented to participate in the study (Fig. 1). At inclusion, median age was 42.5 years compared to 42.0 years $(p=0.22)$ in the remaining WLWH 


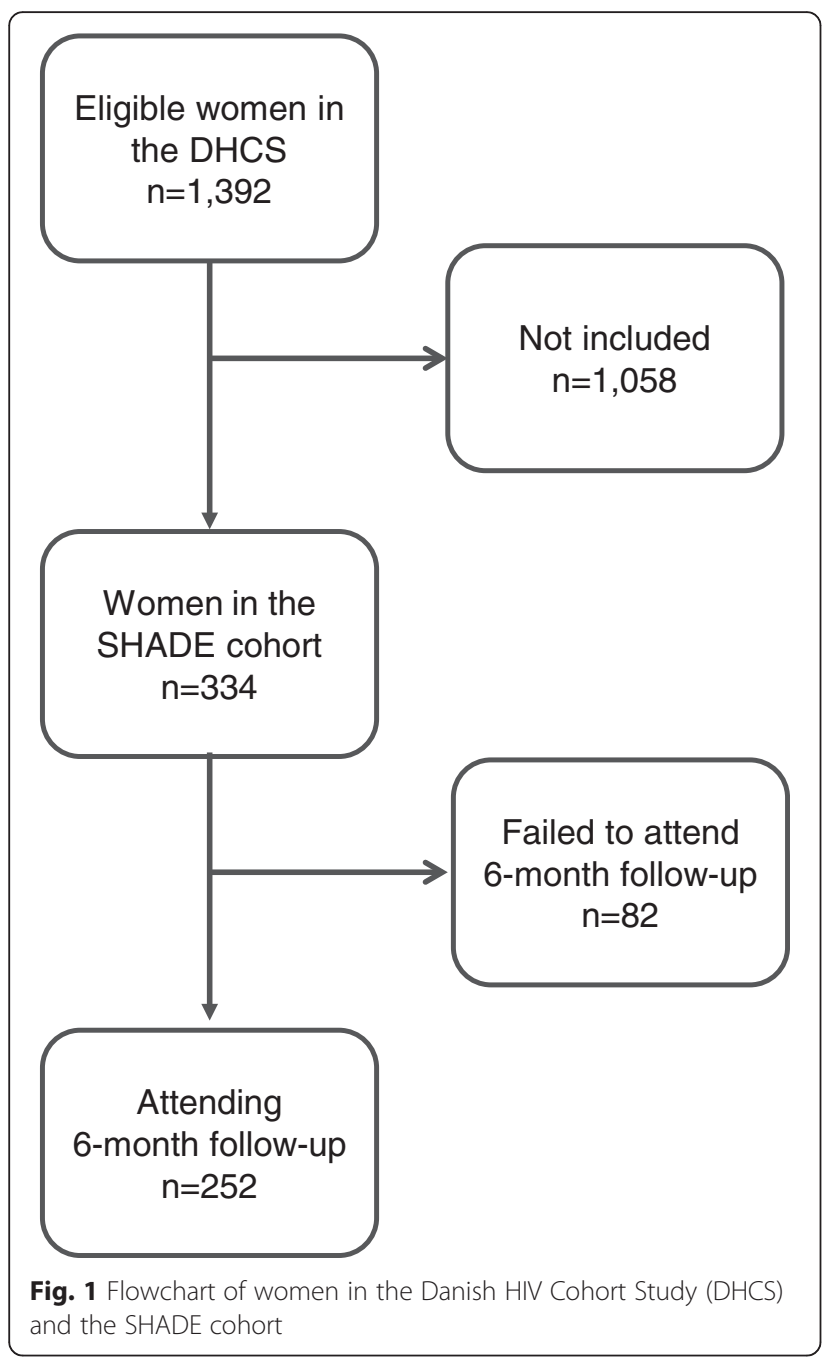

in the DHCS and HIV duration was 11.3 years compared to 10.6 years $(p=0.097)$ (Table 1$)$. Other baseline characteristics are presented in Table 1.

\section{Sexually transmitted infections}

At entry, four (1 \%) women presented with CT (Table 1). Two of these reported condom use and two had lower abdominal symptoms. No participants tested positive for NG (Table 1). Positive serologic syphilis tests were found in eight (2\%) participants; all were interpreted as past infections; 7 (88 \%) of these women were immigrants (Table 1). A history of genital herpes infection was stated by 64 (19\%). No participants were HSV-1 PCR positive, but six (2\%) tested HSV-2 positive by PCR; of whom all were on HAART (Table 1). The prevalence of STIs was too low to perform adjusted analyses aiming at predicting associated factors.

\section{Use of contraceptives - 6 month follow-up}

A total of 252 (76\% of the SHADE cohort) women attended the 6 month follow-up (Fig. 1). Median time from inclusion to follow-up was median 6.2 (IQR 5.9-7.4) months. At follow-up, 168 (69\%) reported sexual activity in the past 6 months - of these 165 (65\%) responded to the question regarding contraceptive use (Table 2). Overall, 124 (75\%) of the sexual active women used contraceptives (Table 2). Among the 41 women using no contraceptives, 29 were of reproductive age ( $<50$ years) and 10 of these stated a desire for pregnancy - leaving 19 (13\% of the sexually active WLWH in the reproductive age) without birth control.

Condom use alone or as part of dual protection were stated by 102 (62\%) participants (Table 2). Having an HIV-negative partner predicted condom use (OR 3.89 (95\% CI 1.49-10.11)) (Table 3). A sensitivity analysis of the adjusted analysis was performed to check for the effect of missing values on outcome by adding an extra category with missing values. This had no impact on the estimates.

Fifteen (9\%) of the sexually active women reported use of hormonal contraception $(\mathrm{HC})$ alone or as part dual protection; of these; 14 were on HAART and 13 (93\%) received a regimen containing either efavirenz, nevirapine, etravirine, atazanavir/ritonavir or darunavir/ ritonavir with possible drug-drug interactions with $\mathrm{HC}$.

\section{Discussion}

In this multicenter, prospective, observational cohort of WLWH in Denmark, we found a low prevalence of STIs thus questioning the need for annual STI screening in WLWH as recommended by e.g. the EACS guidelines [6]. The majority of sexually active WLWH used condoms; primarily when being in a discordant relationship. More than $10 \%$ of sexually active WLWH in the reproductive age were at risk of unintended pregnancy. Finally, the majority of WLWH on both HAART and HC received a combination with potential drug-drug interactions that could decrease contraceptive efficacy.

WLWH in SHADE was no different from WLWH in the remaining nationwide DHCS with regards to age, HIV and race, however they were more likely to be sexually infected with HIV, having a higher CD4 count and a greater probability of being on HAART with a suppressed viral load. We attribute these differences to the exclusion criteria regarding drug abuse.

The viral load of HIV in plasma is a major determinant of HIV transmission [15] and the correlation between plasma and genital viral load is strong $[1,15]$. Genital HIV shedding is also influenced by local factors such as genital ulcers, CT, NG and HSV [5, 15-17]. The increased incidences of STIs in Western cohorts of PLHIV [3, 4] is therefore of concern. Especially, asymptomatic STIs are 
Table 1 Baseline characteristics and prevalence of sexually transmitted infections in women living with HIV (WLWH) in the study and WLWH in the remaining Danish HIV Cohort Study (DHCS)

\begin{tabular}{|c|c|c|c|}
\hline & WLWH in the study & WLWH in the remaining DHCS & $p$-value \\
\hline Number of individuals & $334(24.0)$ & $1,058(76.0)$ & $N A^{1}$ \\
\hline Follow-up (years), median (IQR) & $11.3(5.9-16.9)$ & $10.6(5.5-15.9)$ & 0.097 \\
\hline Follow-up time, total (person-years) & 3,853 & 11,183 & $N A^{1}$ \\
\hline Age at inclusion, median (IQR), (years) & $42.5(36.8-48.3)$ & $42.0(35.5-48.2)$ & 0.22 \\
\hline \multicolumn{4}{|l|}{ Race, n (\%) } \\
\hline White & $141(42.6)$ & $398(39.5)$ & \multirow[t]{5}{*}{$0.041^{2}$} \\
\hline Asian & $44(13.3)$ & $114(11.3)$ & \\
\hline Black & $143(43.2)$ & $461(45.7)$ & \\
\hline \multirow[t]{2}{*}{ Other (missing) } & $3(0.9)$ & $35(3.5)$ & \\
\hline & (3) & (50) & \\
\hline Place of HIV transmission, n (\%) & $114(37.8)$ & $326(34.8)$ & \multirow[t]{6}{*}{0.11} \\
\hline Denmark & $27(8.9)$ & $77(8.2)$ & \\
\hline Europe + US & $128(42.4)$ & $416(44.4)$ & \\
\hline Africa & $33(10.9)$ & $101(10.8)$ & \\
\hline Asia & $0(0)$ & $17(1.8)$ & \\
\hline Other (missing) & $(32)$ & $(121)$ & \\
\hline \multicolumn{4}{|l|}{ Mode of transmission, n (\%) } \\
\hline Heterosexual & $294(91.6)$ & 799 (83.6) & \multirow[t]{4}{*}{0.0015} \\
\hline IDU & $16(5.0)$ & $106(11.1)$ & \\
\hline \multirow[t]{2}{*}{ Other (missing) } & $11(3.4)$ & $51(5.3)$ & \\
\hline & (13) & $(102)$ & \\
\hline \multicolumn{4}{|l|}{ 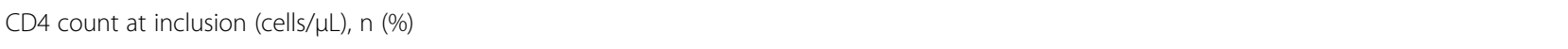 } \\
\hline$<200$ & $12(3.9)$ & $79(9.1)$ & \multirow[t]{4}{*}{0.012} \\
\hline $200-350$ & $51(16.5)$ & $138(15.9)$ & \\
\hline$>350$ & $247(79.7)$ & $650(75.0)$ & \\
\hline (missing) & $(24)$ & $(191)$ & \\
\hline \multicolumn{4}{|l|}{ HAART at inclusion), n (\%) } \\
\hline Yes & $317(94.9)$ & $866(81.8)$ & \multirow[t]{3}{*}{$<0.0001$} \\
\hline No & $17(5.1)$ & $192(18.2)$ & \\
\hline (missing) & $(0)$ & (0) & \\
\hline \multicolumn{4}{|c|}{ On HAART with HIV RNA < 40 copies/mL), n (\%) } \\
\hline Yes & $250(83.6)$ & $576(75.5)$ & \multirow[t]{3}{*}{0.0042} \\
\hline No & $49(16.4)$ & $187(24.5)$ & \\
\hline (missing) & $(18)$ & $(103)$ & \\
\hline \multicolumn{4}{|l|}{ Lifetime sexual partners, n (\%) } \\
\hline$<5$ & 99 (29.6) & -3 & \multirow[t]{6}{*}{$N A^{1}$} \\
\hline $5-14$ & $135(40.4)$ & & \\
\hline $15-25$ & $45(13.5)$ & & \\
\hline$>25$ & $53(15.9)$ & & \\
\hline Does not wish to respond & $2(0.6)$ & & \\
\hline (missing) & (0) & & \\
\hline
\end{tabular}


Table 1 Baseline characteristics and prevalence of sexually transmitted infections in women living with HIV (WLWH) in the study and WLWH in the remaining Danish HIV Cohort Study (DHCS) (Continued)

\begin{tabular}{|c|c|c|c|}
\hline \multicolumn{4}{|l|}{ History of genital herpes infection, $\mathrm{n}(\%)$} \\
\hline Yes & $64(19.2)$ & \multirow[t]{4}{*}{-3} & \multirow[t]{4}{*}{$N A^{1}$} \\
\hline No & $227(68.0)$ & & \\
\hline Unknown & $43(12.8)$ & & \\
\hline (missing) & $(0)$ & & \\
\hline \multicolumn{4}{|l|}{ Symptoms from the lower abdomen, n (\%) } \\
\hline Yes & $78(23.3)$ & \multirow[t]{4}{*}{$--^{3}$} & \multirow[t]{4}{*}{$N A^{1}$} \\
\hline No & $237(71.0)$ & & \\
\hline Does not wish to respond & $19(5.7)$ & & \\
\hline (missing) & $(0)$ & & \\
\hline \multicolumn{4}{|c|}{ Outline of specific symptoms from the lower abdomen, n (\% of women stating "yes" to lower abdominal symptoms) } \\
\hline Vaginal discharge & $30(38.5)$ & \multirow[t]{7}{*}{-3} & \multirow[t]{7}{*}{$N A^{\prime}$} \\
\hline Burning sensation when urinating & $8(10.3)$ & & \\
\hline Abnormal menstrual bleeding & $24(30.8)$ & & \\
\hline Bleeding during sexual intercourse & $6(7.7)$ & & \\
\hline Pain while at rest & $9(11.5)$ & & \\
\hline Pain during sexual intercourse & $15(19.2)$ & & \\
\hline Other & $34(43.6)$ & & \\
\hline Chlamydia trachomatis infection, $\mathrm{n}(\%)^{4,5}$ & $4(1.2)$ & \multirow[t]{3}{*}{-3} & \multirow[t]{3}{*}{$N A^{1}$} \\
\hline Cervical & 3 & & \\
\hline Urethral & 3 & & \\
\hline Neisseria gonorrhoeae infection, $\mathrm{n}(\%)^{6}$ & $0(0)$ & \multirow[t]{5}{*}{-3} & \multirow[t]{5}{*}{$N A^{1}$} \\
\hline Cervical & 0 & & \\
\hline Urethral & 0 & & \\
\hline Rectal & 0 & & \\
\hline Pharyngeal & 0 & & \\
\hline \multicolumn{4}{|l|}{ Syphilis, n (\%) } \\
\hline Any positive serologic test & $8(2.5)$ & \multirow[t]{2}{*}{-3} & \multirow[t]{2}{*}{$N A^{1}$} \\
\hline Positive serology requiring treatment & $0(0)$ & & \\
\hline HSV-1 infection, n (\%) ${ }^{8,9}$ & $0(0)$ & -3 & $N A^{1}$ \\
\hline HSV-2 infection, $n(\%)^{8,9}$ & $6(1.9)$ & -3 & $N A^{1}$ \\
\hline
\end{tabular}

worrying due to complications related to the STI in question and rise in HIV infectiousness [5]. In WLWH, however, data on STIs, contraceptive choices, and sexual health are scarce $[4,8]$.

\section{Sexually transmitted infections}

Only four (1\%) participants presented with $C T$, two of whom had symptoms. No participants were diagnosed with NG. In comparison, the point prevalence of $C T$ and $N G$ in WLWH has been reported to be 1-27 \% in studies carried out in the Western setting, with the highest prevalence found in cohorts of young, newly diagnosed WLWH [5, 18]. The long median HIV duration of 11 years in the present cohort impedes comparison of these studies. Interestingly, an older Danish study on the general population found a comparable prevalence of $C T<2 \%$ in women older than 32 years of age and $<1 \%$ in women older than 43 years of age [19].

The increasing rates of syphilis in MSM living with HIV suggest a sexual high-risk behaviour [3] that is not prevalent amongst WLWH in this study. Whereas more than $70 \%$ of women reported $<15$ lifetime sexual 
Table 2 Characteristics on marital status, sexual activity and contraception at 6-month follow-up $(n=252)$

\begin{tabular}{|c|c|}
\hline & WLWH in the study \\
\hline \multicolumn{2}{|l|}{ Marital status, n (\%) } \\
\hline Married & $105(43.0)$ \\
\hline Cohabitating & $35(14.3)$ \\
\hline Regular partner (not cohabitating) & $27(11.1)$ \\
\hline Single & 77 (31.6) \\
\hline (missing) & (8) \\
\hline \multicolumn{2}{|l|}{ Partner's HIV status, n (\%) } \\
\hline HIV-positive & $50(29.1)$ \\
\hline HIV-negative & $118(68.6)$ \\
\hline Not tested/do not know & $4(2.3)$ \\
\hline (missing) & $(80)$ \\
\hline \multicolumn{2}{|l|}{ Sexual activity in the past 6 months, $\mathrm{n}(\%)$} \\
\hline Yes & $168(68.6)$ \\
\hline No & $77(31.4)$ \\
\hline (missing) & $(7)$ \\
\hline \multicolumn{2}{|c|}{ Current use of contraception in sexually active women, $\mathrm{n}(\%)$} \\
\hline Condom & $84(50.9)$ \\
\hline Condom + hormonal contraception & $6(3.6)$ \\
\hline Condom + intra uterine device & $1(0.6)$ \\
\hline Condom + sterilization & $11(6.7)$ \\
\hline Hormonal contraception & $9(5.5)$ \\
\hline Intra uterine device & $5(3.0)$ \\
\hline Sterilization & $6(3.6)$ \\
\hline Other & $2(1.2)$ \\
\hline Nothing & $41(24.9)$ \\
\hline (missing) & (3) \\
\hline \multicolumn{2}{|c|}{ Reasons for no condom use in sexually active women, $n(\%)^{1,2}$} \\
\hline Patient's wish & $2(3.2)$ \\
\hline Partner's wish & $11(17.7)$ \\
\hline Joint decision & $25(40.3)$ \\
\hline Partner has HIV & $23(37.1)$ \\
\hline Attempts to become pregnant & $10(16.1)$ \\
\hline HIV RNA is low & $13(21.0)$ \\
\hline
\end{tabular}

${ }^{1}$ More than one answer allowed, $\mathrm{n}(\%)$ does not add up to $100 \%,{ }^{2} \mathrm{~N}(\%$ of women responding to the specific question)

partners, this number is markedly higher in MSM cohorts [20]. Also, compared to international female HIV cohorts the number is low; in the Women's Interagency HIV Study (WIHS) $31 \%$ had 10-49 lifetime sexual partners and $24 \%$ reported $>50$ sexual partners [21]. The prevalence of syphilis in cohorts of WLWH in Western settings is $2-13 \%$ - peaking amongst newly diagnosed WLWH [5]. In the present study no syphilis cases were seen, however, the majority of WLWH presenting with positive syphilis serology indicating a prior infection were immigrants, which suggests a lower threshold for screening in this subgroup.

Up to half of all newly diagnosed PLHIV demonstrate HSV-2 infection, which has been ascribed to the association between ulcerative STIs and HIV [22]. Here, more than $40 \%$ of participants reported to be infected with HIV in Africa, where HIV and HSV co-infection is highly prevalent [23]. Yet, no women were tested positive by PCR of HSV-1 and only $2 \%$ tested PCR positive for HSV-2, while about one fifth reported a history of genital herpes infection. All participants shedding HSV2 were on HAART, which is in line with studies finding no attenuation of HSV-2 reactivation in individuals on suppressive HAART [24]. In studies of HSV-2 seropositive WLWH, a markedly higher prevalence of $11-23 \%$ of HSV-2 shedding were found [24, 25].

\section{Use of contraceptives}

Studies on contraceptive use in WLWH are strikingly scarce $[8,26]$. The proportion of participants reporting use of any contraceptive method was higher in the study group, than the estimated proportion of contraceptive use in developed regions of the world and the global proportion in 2011; $75 \%, 70 \%$ and $63 \%$, respectively [27]. However, $>10 \%$ of sexually active participants were in the reproductive age, had no pregnancy desire, and nonetheless used no birth control.

Though, the number of lifetime sexual partners was low compared to the WIHS, the number of women reporting to be sexually active in the past 6 months was comparable at around $70 \%$ [21]. Preferred contraceptives were condoms used by $62 \%$ of sexually active women. In comparison, $26-80 \%$ reported using a barrier contraceptive method at least intermittently in cohorts of young PLHIV $[18,28,29]$. Condom use in the present study was predicted by having an HIV-negative partner.

The metabolism of both $\mathrm{HC}$ and some non-nucleoside reverse-transcriptase inhibitors (NNRTIs) and protease inhibitors (PIs) is dependent on the cytochrome P450 system in the liver $[7,26]$. The NNRTIs efavirenz and nevirapine both decrease effectiveness of $\mathrm{HC}$ [7], while no dose adjustment is needed for rilpivirine, etravirine can be co-administered with $35 \mu \mathrm{g}$ estrogen [7]. Prescription of PIs boosted with ritonavir is not recommended in combination with $\mathrm{HC}$ with the exception of atazanavir, which however requires a dose of at least $30 \mu \mathrm{g}$ estrogen [7]. In the present study, 13 out of 14 participants on both HAART and HC were on a regimen with possible drug-drug interactions and therefore at risk of reduced contraceptive efficacy.

The strengths of this study include the prospective nature and the use of validated laboratory tests. Additionally, the standardized methods of data collection and the 
Table 3 Unadjusted and adjusted odds ratios for predictors of condom use in sexually active women stating use of contraception at 6-month follow-up $(n=165)$

\begin{tabular}{|c|c|c|c|c|c|c|}
\hline Predictors of condom use & $\begin{array}{l}\text { No condom use } \\
(n=63)\end{array}$ & $\begin{array}{l}\text { Condom use } \\
(n=102)\end{array}$ & Unadjusted odds ratios & $p$-value & $\begin{array}{l}\text { Mutually adjusted } \\
\text { odds ratios }\end{array}$ & $p$-value \\
\hline \multicolumn{7}{|c|}{ Age at 1 February 2011 (inclusion), (years) } \\
\hline$>50$ & $15(57.7)$ & $11(42.3)$ & 1.00 & - & 1.00 & - \\
\hline $30-49$ & $44(34.9)$ & $82(65.1)$ & $2.54(1.08-6.00)$ & 0.034 & $2.30(0.66-7.99)$ & 0.19 \\
\hline $18-29$ & $4(30.8)$ & $9(69.2)$ & $3.07(0.75-12.59)$ & 0.12 & $1.14(0.11-11.54)$ & 0.91 \\
\hline (missing) & (0) & $(0)$ & & & & \\
\hline Combined $p$-value & & & & 0.089 & & 0.36 \\
\hline \multicolumn{7}{|l|}{ Race, n (\%) } \\
\hline White & $27(38.6)$ & $43(61.4)$ & 1.00 & - & 1.00 & - \\
\hline Asian & $11(45.8)$ & $13(54.2)$ & $0.74(0.29-1.89)$ & 0.53 & $0.62(0.19-2.07)$ & 0.44 \\
\hline Black & $24(34.3)$ & $46(65.7)$ & $1.20(0.60-2.40)$ & 0.60 & $0.66(0.25-1.72)$ & 0.39 \\
\hline (missing) & (1) & $(0)$ & & & & \\
\hline Combined $p$-value & & & & 0.60 & & 0.64 \\
\hline \multicolumn{7}{|l|}{ HIV RNA at inclusion, $\mathrm{n}(\%)^{2}$} \\
\hline Undetectable & $45(35.2)$ & $83(64.8)$ & 1.00 & - & 1.00 & - \\
\hline Detectable & $13(54.2)$ & $11(45.8)$ & $0.46(0.19-1.11)$ & 0.083 & $0.86(0.30-2.49)$ & 0.78 \\
\hline (missing) & (5) & (8) & & & & \\
\hline Combined $p$-value & & & & 0.083 & & 0.78 \\
\hline \multicolumn{7}{|c|}{ Lifetime sexual partners at inclusion, $\mathrm{n}(\%)$} \\
\hline$<4$ & $22(47.8)$ & $24(52.2)$ & 1.00 & - & 1.00 & - \\
\hline $5-14$ & $25(36.8)$ & $43(63.2)$ & $1.58(0.74-3.37)$ & 0.24 & $1.40(0.52-3.79)$ & 0.51 \\
\hline $15-25$ & $9(34.6)$ & $17(65.4)$ & $1.73(0.64-4.68)$ & 0.28 & $0.94(0.23-3.80)$ & 0.93 \\
\hline$>25$ & $7(28.0)$ & $18(72.0)$ & $2.36(0.83-6.72)$ & 0.11 & $0.96(0.23-4.00)$ & 0.96 \\
\hline (missing) & (0) & (0) & & & & \\
\hline Combined $p$-value & & & & 0.38 & & 0.85 \\
\hline \multicolumn{7}{|l|}{ Marital status, n (\%) } \\
\hline Married & $35(41.2)$ & $50(58.8)$ & 1.00 & - & 1.00 & - \\
\hline Cohabitating & $17(53.1)$ & $15(46.9)$ & $0.62(0.27-1.40)$ & 0.25 & $0.55(0.20-1.50)$ & 0.24 \\
\hline Regular partner (not cohabitating) & $8(33.3)$ & $16(66.7)$ & $1.40(0.54-3.63)$ & 0.49 & $1.01(0.31-3.23)$ & 0.99 \\
\hline Single & $3(12.5)$ & $21(87.5)$ & $4.90(1.36-17.70)$ & 0.015 & - & 0.99 \\
\hline (missing) & (0) & $(0)$ & & & & \\
\hline Combined $p$-value & & & & 0.030 & & 0.67 \\
\hline \multicolumn{7}{|l|}{ Partner's HIV status, n (\%) } \\
\hline HIV-positive & $30(68.2)$ & $14(31.8)$ & 1.00 & - & 1.00 & - \\
\hline HIV-negative & $27(28.7)$ & $67(71.3)$ & $5.32(2.45-11.55)$ & $<0.000$ & $3.89(1.49-10.11)$ & 0.0054 \\
\hline Not tested/do not know & $2(50.0)$ & $2(50.0)$ & $2.14(0.27-16.81)$ & 1 & $1.01(0.05-19.87)$ & 0.99 \\
\hline (missing) & (4) & (19) & & 0.47 & & \\
\hline Combined $p$-value & & & & 0.0001 & & 0.014 \\
\hline \multicolumn{7}{|c|}{ Selfreported HSV ${ }^{3}$ infection prior to inclusion, n (\%) } \\
\hline Yes & $14(43.8)$ & $18(56.2)$ & 1.00 & - & 1.00 & - \\
\hline No & $40(36.0)$ & $71(64.0)$ & $1.38(0.62-3.07)$ & 0.43 & $1.75(0.66-4.63)$ & 0.26 \\
\hline Do not know (missing) & $9(40.9)$ & $13(59.1)$ & $1.12(0.37-3.38)$ & 0.84 & $1.63(0.43-6.25)$ & 0.47 \\
\hline Combined $p$-value & & & & 0.70 & & 0.53 \\
\hline
\end{tabular}

\footnotetext{
${ }^{1}$ The validity of the model was tested using the Hosmer and Lemeshow Goodness-of-Fit Test, ${ }^{2} \mathrm{HIV}-\mathrm{RNA}<40$ copies $/ \mathrm{ml},{ }^{3} \mathrm{HSV}=$ Herpes simplex virus infection
} 
performance of double manual data entry enhanced accuracy of data input and integrity of the interview results. Lastly, the ability to link to the nationwide, complete DHCS with basic data on all PLHIV in Denmark optimises results.

Limitations of the study were that women were only recruited on days where nurses or doctors responsible of the study were present in the outpatient clinic. However, due to the DHCS differences between groups can be addressed. Though, we present a low point prevalence of STIs, incidence of STIs in this cohort has not been examined. Finally, self-reported data especially on sexual history may be unreliable [30].

\section{Conclusion}

Overall, the prevalence of STIs in WLWH in Denmark was low. Screening for STIs at entry of HIV care may prove valuable as shown by other cohorts [5] and due to the fact that especially immigrants presented with positive syphilis serology indicating prior infection. However, annual screening for STIs in WLWH attending routine HIV care is of questionable value. Three quarters of sexually active WLWH used contraception. Condoms were preferred contraceptives, especially when having an HIV-negative partner. The majority of WLWH receiving both HAART and $\mathrm{HC}$ were on a regimen with possible drug-drug interactions with ensuing risk of decreased contraceptive efficacy. The discrepancy between the low rates of STIs and the rather low proportion of condom use can probably be explained by the relatively low number of lifetime sexual partners in this cohort. In our opinion, there is an unmet need for contraceptive counselling in WLWH.

\section{Additional files}

Additional file 1: Questionnaire 1. (DOC $126 \mathrm{~kb})$

Additional file 2: Questionnaire 2. (DOC $123 \mathrm{~kb}$ )

\section{Abbreviations}

Cl: confidence interval; CT: chlamydia trachomatis; CRS: Civil Registration System; DHCS: Danish HIV Cohort Study; HAART: highly active antiretroviral therapy; HC: hormonal contraception; HPV: human papillomavirus; HSV: Herpes simplex virus; MSM: men who have sex with men; NG: Neisseria gonorrhoeae; NNRTI: non-nucleoside reverse-transcriptase inhibitor; NRTI: nucleoside reverse-transcriptase inhibitor; OR: odds ratio; PI: protease inhibitor; PIN: personal identification number; PLHIV: people living with HIV; SHADE: Study on HIV, cervical Abnormalities and infections in women in Denmark; SSI: Statens Serum Institut (the National Institute for Health Data and Diseases Control); STI: sexually transmitted infections; WLWH: women living with HIV; WIHS: Women's Interagency HIV Study.

\section{Competing interests}

$K T$ has received research funding from Abbott and honoraria from JanssenCilag and GlaxoSmithKline/Niiv. JB has in the past served as paid advisor or advisory board member to Roche Molecular Systems, Genomica SAU, and BD Diagnostics. He has received honoraria from Roche, Qiagen, Hologic, Genomica and Bd Diagnostics for lectures. HW has received research funding and honoraria from Roche Molecular, Hologic, and Novo Nordic. TLK has received research funding from Roche, Bristol-Myers Squibb, Merck Sharp \& Dohme, GlaxoSmithKlineNiiv, Abbott, Boehringer Ingelheim, Janssen-Cilag, and Swedish Orphan. AML has received research funding from Abbott and honoraria from Bristol-Myers Squibb, Merck Sharp \& Dohme, GlaxoSmithKline, Boehringer Ingelheim and Janssen-Cilag. SL, MS, FR, IJ, GP, LNN, and NO report no conflicts of interest.

\section{Authors' contributions}

KT contributed to conception and design of the study, included patients, performed interviews and gynaecological examinations, analysed and interpreted data, and drafted the manuscript. SL, biostatistician, was involved in analysis and interpretation of data, and critically reviewed the manuscript. MS contributed to conception and design of the study, included patients, performed interviews and gynaecological examinations, and critically reviewed the manuscript. TLK contributed to conception and design of the study, included patients, performed interviews and gynaecological examinations, and critically reviewed the manuscript. FR included patients, performed interviews and gynaecological examinations, and critically reviewed the manuscript. ISJ contributed to conception and design of the study, included patients, performed interviews and gynaecological examinations, and critically reviewed the manuscript. GP contributed to conception and design of the study, included patients and performed interviews, and critically reviewed the manuscript. LNN included patients and performed interviews, and critically reviewed the manuscript. JB was in charge of the analyses of HSV-1 and 2, and critically reviewed the manuscript. $\mathrm{HW}$ was in charge of the analyses of $C T$ and $N G$, and critically reviewed the manuscript. NO, head of the DHCS, critically reviewed the manuscript. AML, principal investigator, contributed to conception and design of the study, included patients and performed interviews and gynaecological examinations, was involved in analysis and interpretation of data, and critically reviewed the manuscript. All authors read and approved the final manuscript.

\section{Acknowledgements}

We are first and foremost grateful to all the women living with HIV for participating in the study. We further thank the staff of our clinical departments for their continuous support and enthusiasm at the participating centres in the SHADE cohort: Department of Infectious Diseases, Hvidovre, Copenhagen University Hospital (AM Lebech, K Thorsteinsson); Department of Infectious Diseases, Copenhagen University Hospitals, Rigshospitalet (TL Katzenstein, FF Rönsholt); Department of Infectious Diseases, Odense University Hospital (IS Johansen); Department of Infectious Diseases, Aarhus University Hospitals, Skejby (M Storgaard); Department of Infectious Diseases, Aalborg University Hospital (G Pedersen); Department of Infectious Diseases, Hillerød Hospital (LN Nielsen).

Financial support from the Danish Medical Women's Association (Grant of Agnethe Løvgreen), the Aragon Foundation, the Foundation of Aase and Ejnar Danielsens, and the Foundation of Anna and Preben Simonsen are gratefully acknowledged. The sponsors of the study had no role in study design, data collection, data analysis, data interpretation or writing of the article.

\section{Author details}

'Department of Infectious Diseases, Hvidovre, Copenhagen University Hospital, Copenhagen, Denmark. ${ }^{2}$ Clinical Research Center, Hvidovre, Copenhagen University Hospital, Hvidovre, Denmark. ${ }^{3}$ Department of Infectious Diseases, Skejby, Aarhus University Hospital, Aarhus, Denmark. ${ }^{4}$ Department of Infectious Diseases, Copenhagen University Hospital, Rigshospitalet, Copenhagen, Denmark. ${ }^{5}$ Department of Infectious Diseases, Odense University Hospital, Odense, Denmark. ${ }^{6}$ Department of Infectious Diseases, Aalborg University Hospital, Aalborg, Denmark. ${ }^{7}$ Department of Infectious Diseases, Hillerød Hospital, Hillerød, Denmark. ${ }^{8}$ Department of Pathology, Hvidovre, Copenhagen University Hospital, Copenhagen, Denmark. ${ }^{9}$ Institute of Clinical Medicine, University of Copenhagen, Copenhagen, Denmark. ${ }^{10}$ Department of Clinical Microbiology, Hvidovre, Copenhagen University Hospital, Copenhagen, Denmark.

Received: 18 September 2015 Accepted: 2 February 2016

Published online: 16 February 2016 


\section{References}

1. Cohen MS, Chen YQ, McCauley M, Gamble T, Hosseinipour MC, Kumarasamy N, et al. Prevention of HIV-1 infection with early antiretroviral therapy. N Engl J Med. 2011;365:493-505.

2. Rodger A, Bruun T, Cambiano V, Lundgren J, et al. HIV Transmission Risk Through Condomless Sex If HIV+ Partner On Suppressive ART: PARTNER Study. Boston: Conference on Retroviruses and Opportunistic Infections (CROI 2014); 2014. Abstract 153LB. CROI 2014 . 3-3-2014. Ref Type: Abstract.

3. Salado-Rasmussen K, Katzenstein TL, Gerstoft J, Cowan SA, Knudsen TB, Mathiesen L, et al. Risk of HIV or second syphilis infection in Danish men with newly acquired syphilis in the period 2000-2010. Sex Transm Infect. 2013;89:372-6.

4. Sprenger K, Evison JM, Zwahlen M, Vogt CM, Elzi MV, Hauser C, et al. Sexually transmitted infections in HIV-infected people in Switzerland: crosssectional study. Peer J. 2014;2:e537. doi:10.7717/peerj.537.eCollection;\%2014.

5. Kalichman SC, Pellowski J, Turner C. Prevalence of sexually transmitted coinfections in people living with HIV/AIDS: systematic review with implications for using HIV treatments for prevention. Sex Transm Infect. 2011;87:183-90.

6. EACS Guidelines version 7.1 November 2014. http://www.eacsociety.org/ files/guidelines_english_71_141204.pdf. Accessed on March 30th 2015. 303-0015. Ref Type: Internet Communication

7. Tittle V, Bull L, Boffito M, Nwokolo N. Pharmacokinetic and pharmacodynamic drug interactions between antiretrovirals and oral contraceptives. Clin Pharmacokinet. 2015;54:23-34.

8. Loutfy MR, Sherr L, Sonnenberg-Schwan U, Walmsley SL, Johnson M, d'Arminio MA. Caring for women living with HIV: gaps in the evidence. J Int AIDS Soc. 2013;16:18509.

9. http://www.dst.dk/da/Statistik/emner/befolkning-ogbefolkningsfremskrivning/folketal.aspx. Accessed on September 14th 2012 Webpage in Danish. 14-9-2012. Ref Type: Internet Communication

10. http://www.ssi.dk/Service/Sygdomsleksikon/H/AIDS\%20-\%20HIV.aspx. Accessed on September 14th 2012. Webpage in Danish. 14-9-2012. Ref Type: Internet Communication

11. Lauritsen JM, Bruus M. EpiData (version 3.1). A comprehensive tool for validated entry and documentation of data. Odense: The EpiData Association; 2003-2005. 11-2-2015. Ref Type: Generic

12. Pedersen CB. The Danish Civil Registration System. Scand J Public Health. 2011;39:22-5.

13. Obel N, Engsig FN, Rasmussen LD, Larsen MV, Omland LH, Sorensen HT. Cohort profile: the Danish HIV cohort study. Int J Epidemiol. 2009;38:1202-6.

14. National surveillance of syphilis, SSI, 2011. Available from: http://www.ssi.dk/ English/News/EPI-NEWS/2012/No\%2037\%20-\%202012.aspx. Accessed on January 19th 2015. 19-1-2015. 19-1-2015. Ref Type: Internet Communication

15. Tanton C, Weiss HA, Le GJ, Changalucha J, Rusizoka M, Baisley K, et al. Correlates of HIV-1 genital shedding in Tanzanian women. PLoS One. 2011; $6: 17480$.

16. Patel P, Borkowf CB, Brooks JT, Lasry A, Lansky A, Mermin J. Estimating peract HIV transmission risk: a systematic review. AIDS. 2014;28:1509-19.

17. Ward $\mathrm{H}$, Ronn M. Contribution of sexually transmitted infections to the sexual transmission of HIV. Curr Opin HIV AIDS. 2010;5:305-10.

18. Setse RW, Siberry GK, Gravitt PE, Moss WJ, Agwu AL, Wheeling JT, et al. Correlates of sexual activity and sexually transmitted infections among human immunodeficiency virus-infected youth in the LEGACY cohort, United States, 2006. Pediatr Infect Dis J. 2011:30:967-73.

19. Westh $\mathrm{H}$, Kolmos HJ. Large-scale testing of women in Copenhagen has not reduced the prevalence of Chlamydia trachomatis infections. Clin Microbiol Infect. 2003;9:619-24.

20. Videla S, Darwich L, Canadas MP, Coll J, Pinol M, Garcia-Cuyas F, et al. Natural history of human papillomavirus infections involving anal, penile, and oral sites among HIV-positive men. Sex Transm Dis. 2013:40:3-10.

21. Massad LS, Xie X, Burk R, Keller MJ, Minkoff H, D'Souza G, et al. Long-term cumulative detection of human papillomavirus among HIV seropositive women. AIDS. 2014;28:2601-8.

22. Barbour JD, Sauer MM, Sharp ER, Garrison KE, Long BR, Tomiyama $H$, et al. HIV-1/HSV-2 co-infected adults in early HIV-1 infection have elevated CD4+ T cell counts. PLoS One. 2007;2:e1080

23. Celum C, Wald A, Lingappa JR, Magaret AS, Wang RS, Mugo N, et al. Acyclovir and transmission of HIV-1 from persons infected with HIV-1 and HSV-2. N Engl J Med. 2010;362:427-39.
24. Pere H, Rascanu A, LeGoff J, Matta M, Bois F, Lortholary O, et al. Herpes simplex virus type 2 (HSV-2) genital shedding in HSV-2-/HIV-1-co-infected women receiving effective combined antiretroviral therapy. Int J STD AIDS 2015

25. Perti T, Nyati M, Gray G, De BG, Selke S, Magaret A, et al. Frequent genital HSV-2 shedding among women during labor in Soweto, South Africa. Infect Dis Obstet Gynecol. 2014;2014:258291.

26. Tseng A, Hills-Nieminen C. Drug interactions between antiretrovirals and hormonal contraceptives. Expert Opin Drug Metab Toxicol. 2013;9:559-72.

27. World Contraceptive Patterns 2013. United Nations. http://www.un.org/en/ development/desa/population/publications/pdf/family/ worldContraceptivePatternsWallChart2013.pdf. Accessed on April 1st 2015. 1-4-2015. 1-4-2015. Ref Type: Internet Communication

28. Brownstein PS, Gillespie SE, Leong T, Chahroudi A, Chakraborty R, CamachoGonzalez AF. The Association of Uncontrolled HIV Infection and Other Sexually-Transmitted Infections in Metropolitan Atlanta Youth. Pediatr Infect Dis J 2014.

29. Hughes A, Hope RL, Nwokolo N, Ward B, Jones R, Von SM, et al. Meeting complex needs: young people with HIV in London. HIV Med. 2013;14:145-

30. DiClemente RJ, Sales JM, Danner F, Crosby RA. Association between sexually transmitted diseases and young adults' self-reported abstinence. Pediatrics. 2011;127:208-13.

\section{Submit your next manuscript to BioMed Central and we will help you at every step:}

- We accept pre-submission inquiries

- Our selector tool helps you to find the most relevant journal

- We provide round the clock customer support

- Convenient online submission

- Thorough peer review

- Inclusion in PubMed and all major indexing services

- Maximum visibility for your research

Submit your manuscript at www.biomedcentral.com/submit 